\title{
Fuzzy Expert System to Evaluate the Quality of Service Provider in the Implementation of E-Government
}

\author{
Munish Gupta \\ Asst. Professor \\ Dept. of Computer Science Apeejay College of Fine \\ Arts, Jalandhar
}

\begin{abstract}
In this paper, a fuzzy expert system has been developed for categorizing service provider in e-government service center environment. On the basis of data to be collected through case studies carried out within service center, the categories has been derived for service providers based on demographic, experience, and behavioral attributes. The fuzzy expert system will assign service provider to the pre-defined categories and provides the corresponding membership values given into the system using fuzzy logic. A prototype system has been proposed and designed in this paper to identify the type service provider based on the demographic, experience and behavioral attributes.
\end{abstract}

\section{Keywords}

Fuzzy Expert System,e-government, Citizen Satisfaction, Intelligent Service Support System.

\section{INTRODUCTION}

E-Governments have received tremendous response world over. The interpretation of e-Government is quite broad and divergent. General definition describes e-Government as the use of information and communication technologies (ICT) to transform government by making it more accessible, effective and accountable [1]. A variety of public services are now delivered online to the e-citizens. E-Government is no longer just an option but a necessity for countries aiming for better governance. E-Government services involve many people such as citizens or users, e-government employees, public administration and political leaders. Each of these people has different objectives, benefits and interests which ultimately affect the success of e-Government. But paramount objective of the e-Government should be to deliver services to citizens of the country with great quality, convenience and less cost.

However, in the last few years the quality of service has become an issue of great concern in e- Government. EGovernments world over are being criticized for not delivering the services for which they were created. Therefore, there must be some mechanism to judge whether they are able to deliver the services as expected. The eGovernment performance evaluation is important for the development of e-Government. Most of the governments are keen on changing the way they function, and find it crucial to adopt a customer focused approach in their governance [2]. Hence, proper assessment of the customer satisfaction gives us learning on the kind of changes needed to be done in eGovernment to make it successful. Unlike quality of products, quality of service is an abstract concept. With respect to public services the assessment of quality has been relatively less studied. Conventional models for quality of service evaluation are too subjective and inaccurate to be applied in the case of e-Government. Therefore, a model is required to

\author{
Jagmohan Mago \\ Asst. Professor \\ Dept. of Computer Science Apeejay College of Fine \\ Arts, Jalandhar
}

study the quality of services of e-Government and that will have significant impact on the improvement offline public services.

This research intend to develop a model for measuring the level of Citizen Satisfaction and for effective engagement between any citizen and service provider within service centre environment using soft computing techniques, which will contribute to the qualitative improvement of the available offline services of the e-Government and will enhance citizen satisfaction. In this paper, a Fuzzy Expert System is proposed for categorizing service providers to that of the pre-defined category. Based on the categorization, the model will display the necessary information required of a particular citizen to the chosen service provider to render the best possible services accordingly.

\section{LITERATURE REVIEW}

In order to develop such a model, a literature survey of quality of service for e-government services is necessary.[4] conducted a synthetic summary of quality dimensions in egovernment services and concluded that there are two main approaches in measuring the quality of e-government services namely introvert approach and extrovert approach. In introvert approach, the quality of delivered services is measured by the back office procedures, leadership of the organization and management's dedication to quality. The extrovert approach relates to availability, usability and security etc. The said paper proposed a model which covers both the introvert and extrovert approach. [3] Developed a holistic evaluation framework COBRAS based on the measurement factors that impact the satisfaction of users with an e-government service. The framework balances the user's cost and risk of engaging with an e-service with the associated benefit and opportunity from such e-service. The proposed framework is demonstrated as a useful tool for evaluating satisfaction of users and the success of e-government services. [5] used Fuzzy Analytic Hierarchy Process to evaluate the performance of eGovernment. This paper presented a fuzzy MCDM method for the determination of the weights of criteria and sub-criteria of evaluation in e-government performance management. The major advantage of this method is that it can be used for both qualitative and quantitative criteria. The results show that the model has the capability to be flexible and apply in egovernment performance application.[6] Described a knowledge based approach that enables the subjective evaluation of portal and e-service quality by users in an adaptive manner. The model for adaptive quality measurement (MAQM) comprises different ontology including concepts regarding quality aspects, questions and questionnaires, portal characteristics and problems encountered by users while using the portal. A prototype 
adaptive questionnaire system (SALT) which uses the MAQM model was also described. The system applies three axes of adaption: based on real-time feedback from users through questionnaires, based on problems encountered by the users, and based on metadata of the pages visited by the user.[2] Proposed a method to assess in an objective manner the change in service quality as a result of e-Government implementation. The authors have studied the automated civic services system implemented in Ahmedabad, India. Using a set of service attributes stated by the users as important in assessing the systems impact, the paper illustrates the proposed methodology using hypothetical data on the relative importance of the various attributes.

[7] Performed an assessment study in a holistic manner which could address the expectations of all the project stakeholders. A broad assessment framework model which could give a direction to the assessment and learning's which can go back into the project is proposed. [8] Studied that there are three kinds of situations that require evaluation in e-government. One is the e-environment; second is evaluating the performance of an e-government programme or project; and third is the overall impact of e-government on general government functioning, economic development and citizen servicing. Accordingly, three respective approaches of evaluation such as: E-readiness assessment of states or region, Hierarchy of measures taken by the e-government programme or project, Overall impact of e-government is required. [9] Concluded that evaluation of e-government should focus on four domains of e-government: e-services, emanagement, e-democracy and e-commerce. Unfortunately, many decision-makers and researchers concentrate only on the provision of electronic services and consider participation as an unnecessary complexity cost factor. [10] Presented a quality ontology that formalizes all the knowledge needed for the realization of a multi-perspective and adaptive evaluation of e-government services. Different perspectives are taken into account and the mappings between them are defined using the presented ontology, enabling a comprehensive and holistic view of e-government service quality.

[11] Selected efficiency, privacy, responsiveness and web assistance as e-service quality dimensions and concluded that e-service quality has a relation with citizen satisfaction considering four dimensions given above of service quality. Use was found to be positively and significantly related to citizen satisfaction. [13] Described the categorization of customer and service advisors in call Centre and then uses a fuzzy expert system framework to assign each customer call and customer service advisor (CSA) with pre-defined category from categorization. After the authors identify the type of customer and advisor, the minimum amount of information which is required by the advisor to serve the customer can be derived and displayed on the screen. The paper defines a loyalty index that measures decrease in customer satisfaction based on complaints within the call Centre which predicts the time period for churn using customer profiles. A long term estimate on the duration of the customer staying with the company can be derived once the profile has been identified.

The literature has been found lacking in the area of quality assessment from citizen's satisfaction view. All these papers don't focus on quality characteristics related to citizen satisfaction while availing the services of e-government through service provider. The existing work don't give due weightage to the citizen satisfaction. Our work seeks to address these gaps by providing a Quality Assessment Model (QAM) that enables performance evaluation of e-government by measuring the citizen satisfaction level and by developing a Fuzzy Expert System which will help in improving citizen satisfaction.

\section{FUZZY EXPERT MODEL}

In this paper, a fuzzy expert system model is proposed for categorizing service provider with the use of MATLAB fuzzy logic toolbox [12]. The model will assign any citizen to any service provider to render the service within the service center. For developing the Fuzzy Expert System, data is collected through semi structured questionnaires for identifying variables for citizens and service providers. Factor analysis and Clustering analysis derived the factors to be used. Fuzzy expert system assigns each service provider to that of the pre-defined category. Based on the categorization, the model will display the necessary information required for a particular combination of citizen and service provider. All the results are to be validated with expert judgment and in simulated environment. The main features of the Fuzzy Expert System developed are as follows:

Any Service Provider and Any Citizen Situation - Any service provider would be able to serve any citizen and will provide good service.

Citizen and Service Provider Categorization - the important attributes such as demographic and experience levels etc. of citizens and service providers are categorized along with behavioral variables which enable the service providers to provide better customer service.

Proper Use of Information -Information about the customer beforehand would enable the service providers to efficiently use this information and will provide better service.

Customized Information - presenting customized information on the screen based on both citizen and service provider categories derived from categorization.

Citizen Handling - with the help of proposed soft computing methodology levels of citizen handling can be improved where citizens are provided with the service they expect from the service provider.

Real Time - A further benefit of using the proposed method is it could be implemented using real time monitoring.

\section{DEVELOPMENT OF FUZZY EXPERT SYSTEM}

The critical factors which are input variables to the Fuzzy Expert System are Professional Competence, Age, and Behaviour. The professional competence is further based on four parameters namely Qualification, Experience, IT Skills and Competence. Qualification refers to the years of education completed. Experience is number of years in similar services. IT skills refer to the experience in using Information and Communication Technologies (ICT). Competence refers to capability of the service provider to handle the citizens and their problems. Each critical Factor is defined by a membership function which helps to take the crisp input values and transform them into degree of membership. 


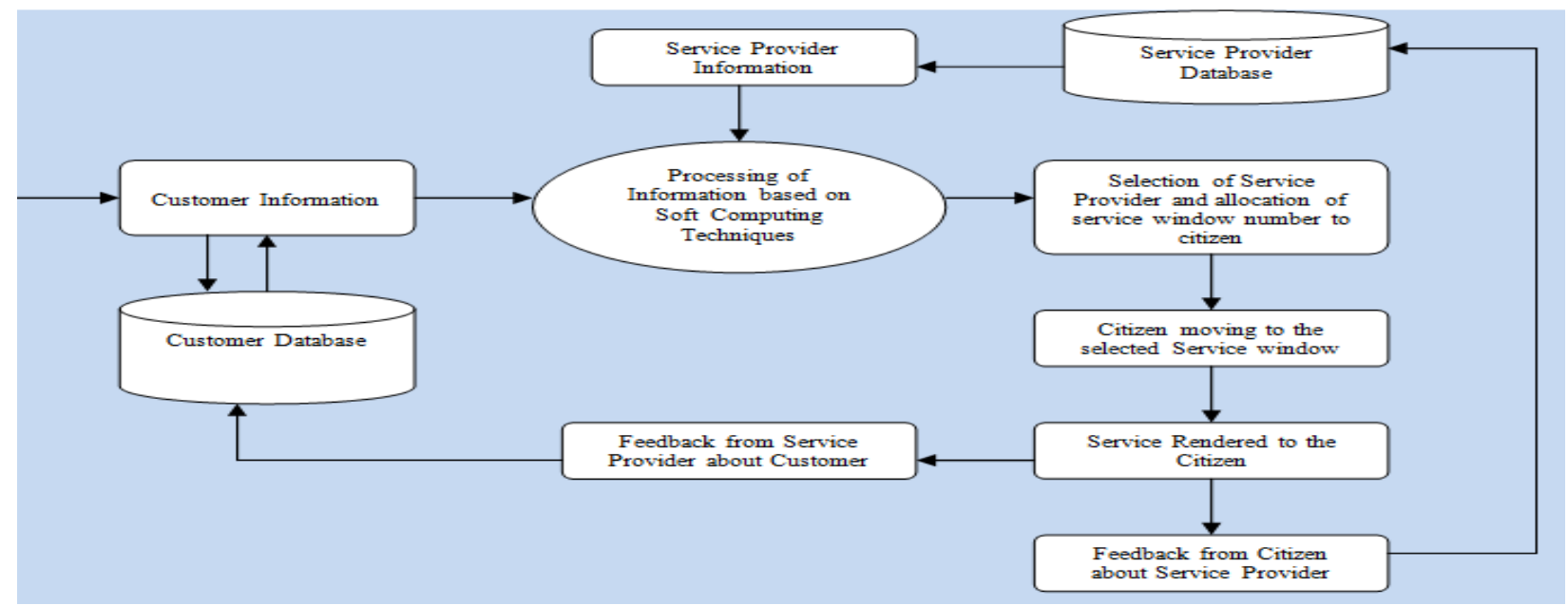

Fig 1: Architecture of Fuzzy Expert System

The trapezoidal membership functions are used which has flat top. The following are the critical factors along with their membership functions which are input to the Fuzzy Expert System.

\subsection{Professional Competence}

Professional Competence is combination of four critical factors namely Qualification, Experience, IT Skills and Competence. The Fig. 2 represents the fuzzy model for the professional competence. The Mamdani's Model is used to compute the professional competence level. Centroid method [14] is used for the defuzzification of the outputs.

$$
x^{*}=\frac{\int \mu_{i}(x) x d x}{\int \mu_{i}(x) d x}
$$

The output of this is the input to the next fuzzy model that evaluates professional competence, age and behavior of the service provider for evaluating overall quality of the service provider.

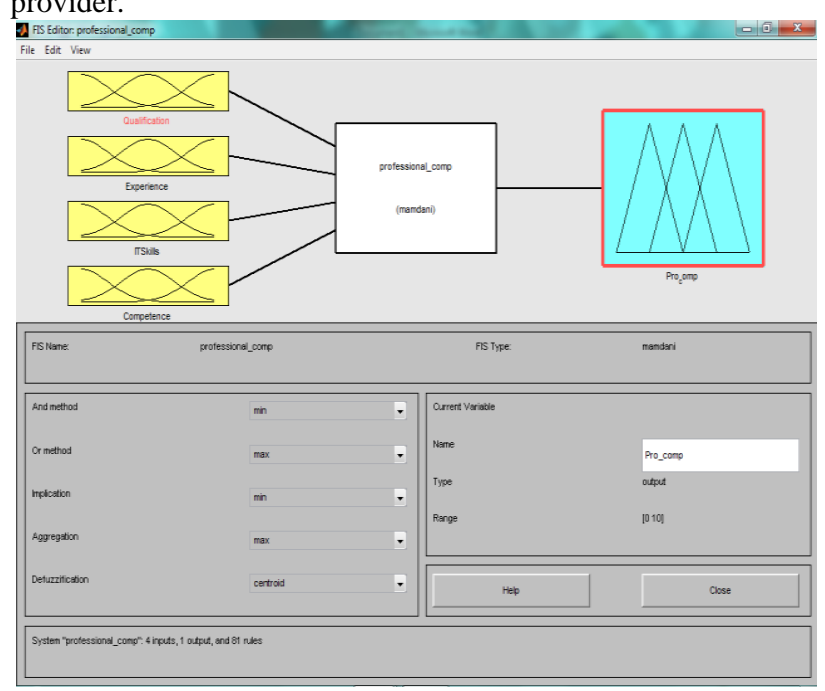

Fig 2: Fuzzy model of level of Professional Competence

\subsubsection{Qualification}

The linguistic terms for the input variable qualification are Primary, School and Graduate and above. The membership function for Qualification is shown in Fig 3.

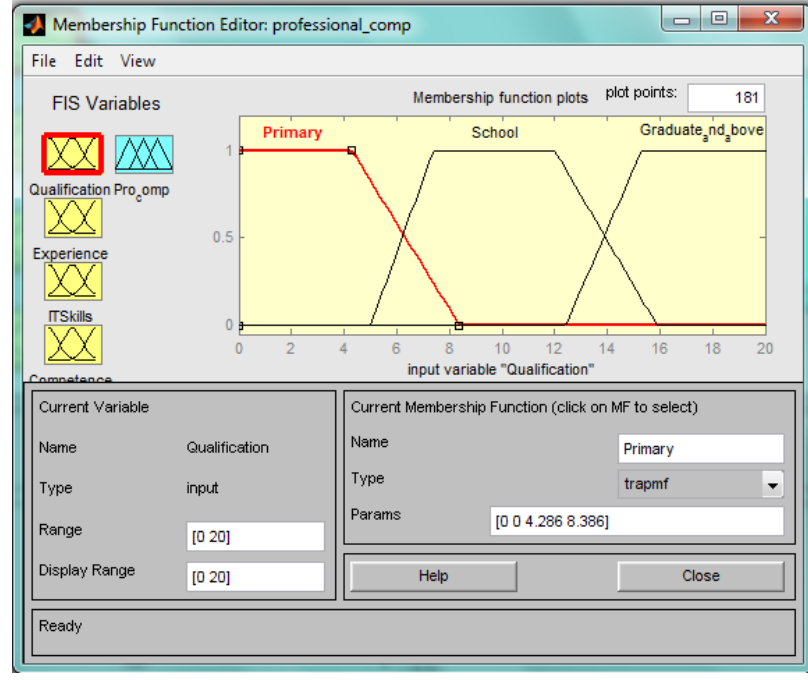

Fig 3: Fuzzy membership functions of Qualification.

\subsubsection{Experience}

The linguistic terms for the input variable Experience are Low, Medium and High. The membership function for Experience is shown in Fig. 4.

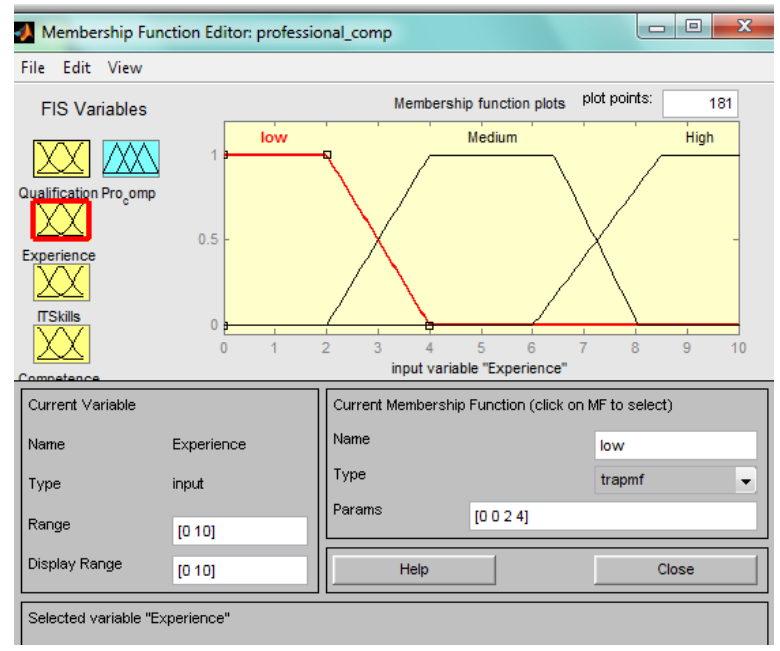

Fig 4: Fuzzy membership functions of Experience. 


\subsubsection{IT Skills}

The linguistic terms for the input variable IT Skills are Low, Medium and High. The membership function for IT Skills is shown in Fig. 5.

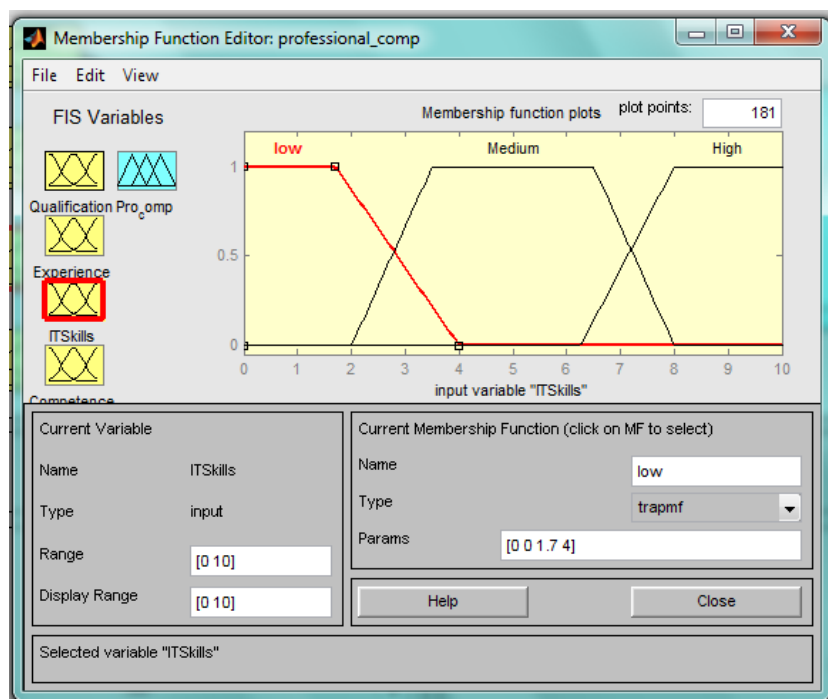

Fig 5: Fuzzy membership functions of IT Skills.

\subsubsection{Competence}

The linguistic terms for the input variable Competence are Low, Medium and High. The membership function for Competence is shown in Fig. 6.

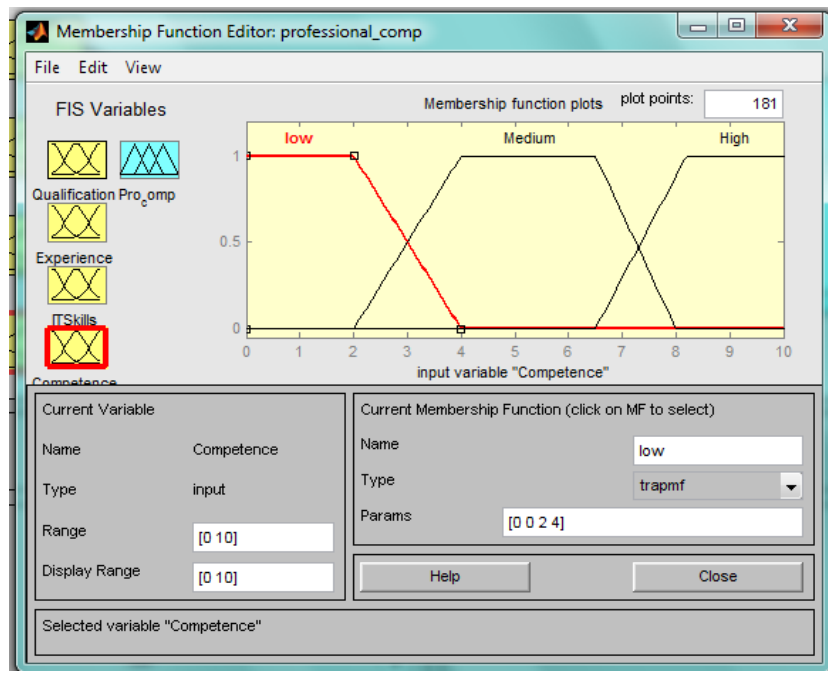

Fig 6: Fuzzy membership functions of Competence.

\subsection{Level of Professional Competence}

Fuzzy Expert System make decisions and generate output values based on the knowledge provided in the form of Fuzzy rules. The Fuzzy rule specifies how the output of the system is determined for various instances of the input variables. The following are the part of Fuzzy Expert System and Fuzzy Rules for categorization of Professional Competence into three categories namely Average, Good and Excellent as show $\mathrm{n}$ in Fig. 7, which is the input to the main Fuzzy Expert System along with Age and Behaviour.

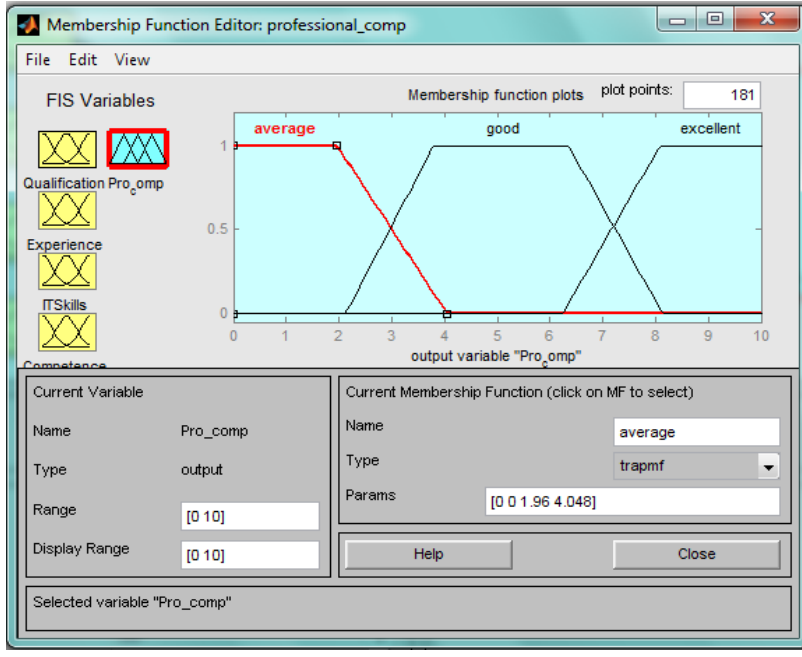

Fig 7: Fuzzy membership functions of Competence Level. (Output)

\subsubsection{Fuzzy Rules for Evaluating Professional} Competence level of Service Provider

1. If (Qualification is Primary) and (Experience is low) and (ITSkills is low) and (Competence is low) then (Pro_comp is average)

2. If (Qualification is Primary) and (Experience is low) and (ITSkills is low) and (Competence is Medium) then (Pro_comp is average)

3. If (Qualification is Primary) and (Experience is low) and (ITSkills is low) and (Competence is High) then (Pro_comp is average)

4. If (Qualification is Primary) and (Experience is low) and (ITSkills is Medium) and (Competence is low) then (Pro_comp is average)

5. If (Qualification is Primary) and (Experience is low) and (ITSkills is Medium) and (Competence is Medium) then (Pro_comp is average)

6. If (Qualification is Primary) and (Experience is low) and (ITSkills is Medium) and (Competence is High) then (Pro_comp is good)

7. If (Qualification is Primary) and (Experience is low) and (ITSkills is High) and (Competence is low) then (Pro_comp is average)

79. If (Qualification is Graduate_and_above) and (Experience is High) and (ITSkills is High) and (Competence is low) then (Pro_comp is excellent)

80. If (Qualification is Graduate_and_above) and (Experience is High) and (ITSkills is High) and (Competence is Medium) then (Pro_comp is excellent)

81. If (Qualification is Graduate_and_above) and (Experience is High) and (ITSkills is High) and (Competence is High) then (Pro_comp is excellent) 


\subsection{Age}

The linguistic terms for the input variable Age are Young, Middle Aged and Old. The membership function for Age is shown in Fig. 8.

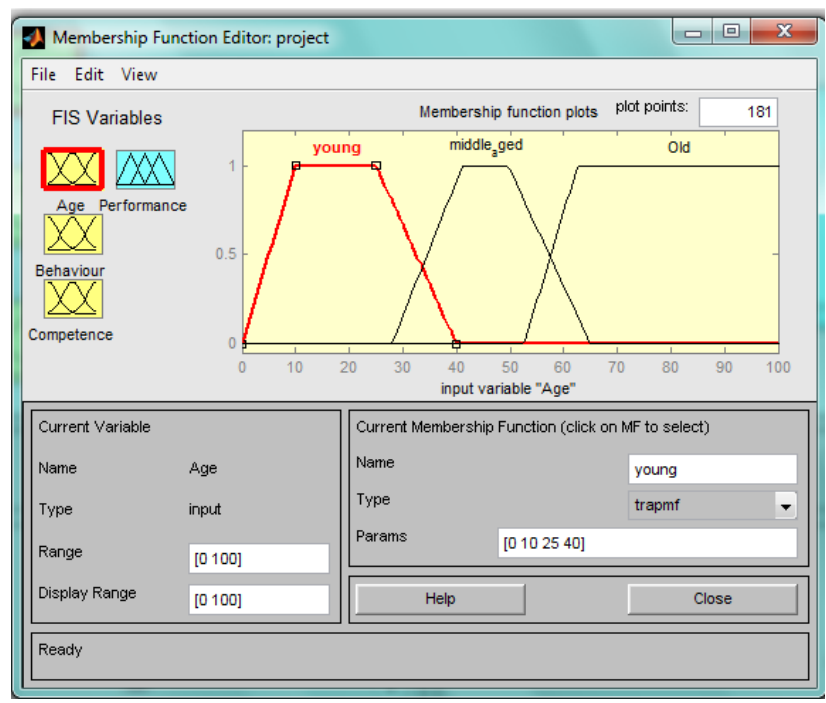

Fig 8: Fuzzy membership functions of Age.

\subsection{Behaviour}

The linguistic terms for the input variable Behaviour are Annoyed, Friendly and Customer Oriented. The membership function for Behaviour is as shown in Fig. 9.

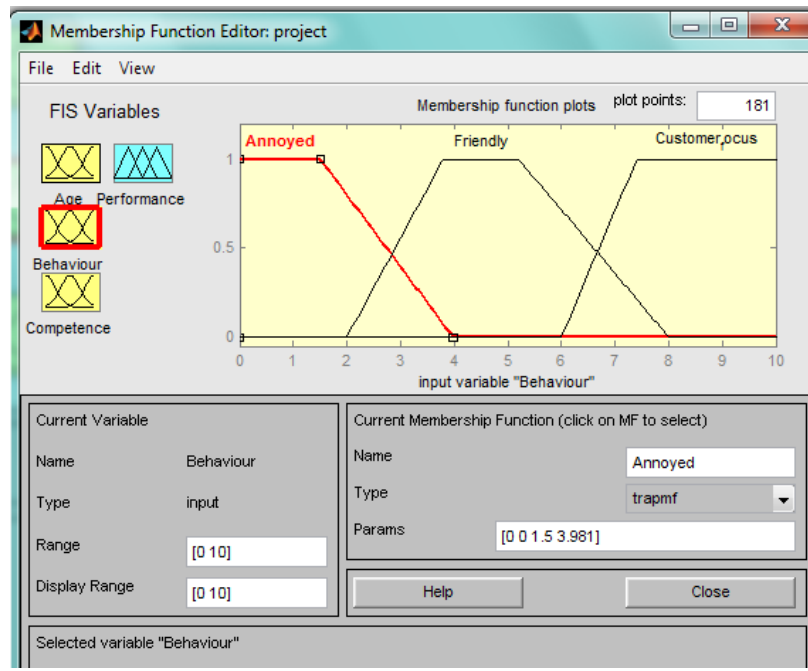

Fig 9: Fuzzy membership functions of Behaviour.

\subsection{Overall Competence Level of Service Provider}

Fig. 10 depicts the output membership function for deciding the overall quality of the service provider. The output depends on the input of three factors namely, age, behaviour and the professional competence. The professional competence is computed in the previous module as shown in Fig. 7. The output of that module is an input to this module. In this research nested fuzzy system has been defined. The following are the Fuzzy Expert System and Fuzzy Rules for categorization of Service Provider into three categories namely Average, Good and Excellent, that represents the quality of the service provided by the service provider.

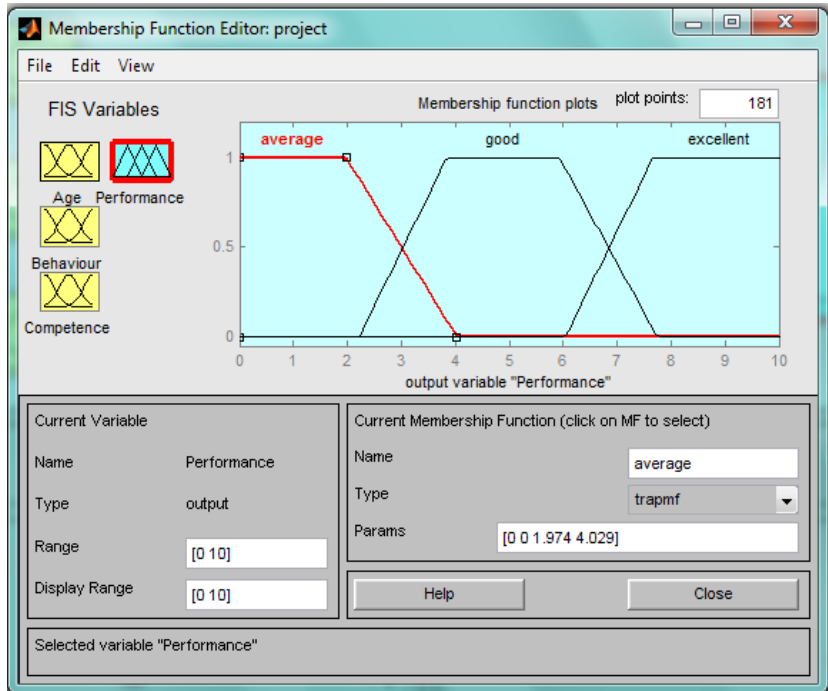

Fig10: Fuzzy membership functions of Overall Competence Level of Service Provider. (Output)

\subsubsection{Sample Rules for Evaluating Overall} Competence level of Service Provider

1. If (Age is young) and (Behaviour is Annoyed) and (Competence is low) then (Performance is average)

2. If (Age is young) and (Behaviour is Annoyed) and (Competence is Medium) then (Performance is average)

3. If (Age is young) and (Behaviour is Annoyed) and (Competence is High) then (Performance is good)

4. If (Age is young) and (Behaviour is Friendly) and (Competence is low) then (Performance is average)

5. If (Age is young) and (Behaviour is Friendly) and (Competence is Medium) then (Performance is average)

6. If (Age is young) and (Behaviour is Friendly) and (Competence is High) then (Performance is excellent)

7. If (Age is young) and (Behaviour is Customer_Focus) and (Competence is High) then (Performance is excellent)

25. If (Age is Old) and (Behaviour is Customer_focus) and (Competence is low) then (Performance is average)

26. If (Age is Old) and (Behaviour is Customer_focus) and (Competence is Medium) then (Performance is good)

27. If (Age is Old) and (Behaviour is Customer_focus) and (Competence is High) then (Performance is excellent) 
QUALITY OF SERVICE PROVIDED BY THE SERVICE PROVIDER

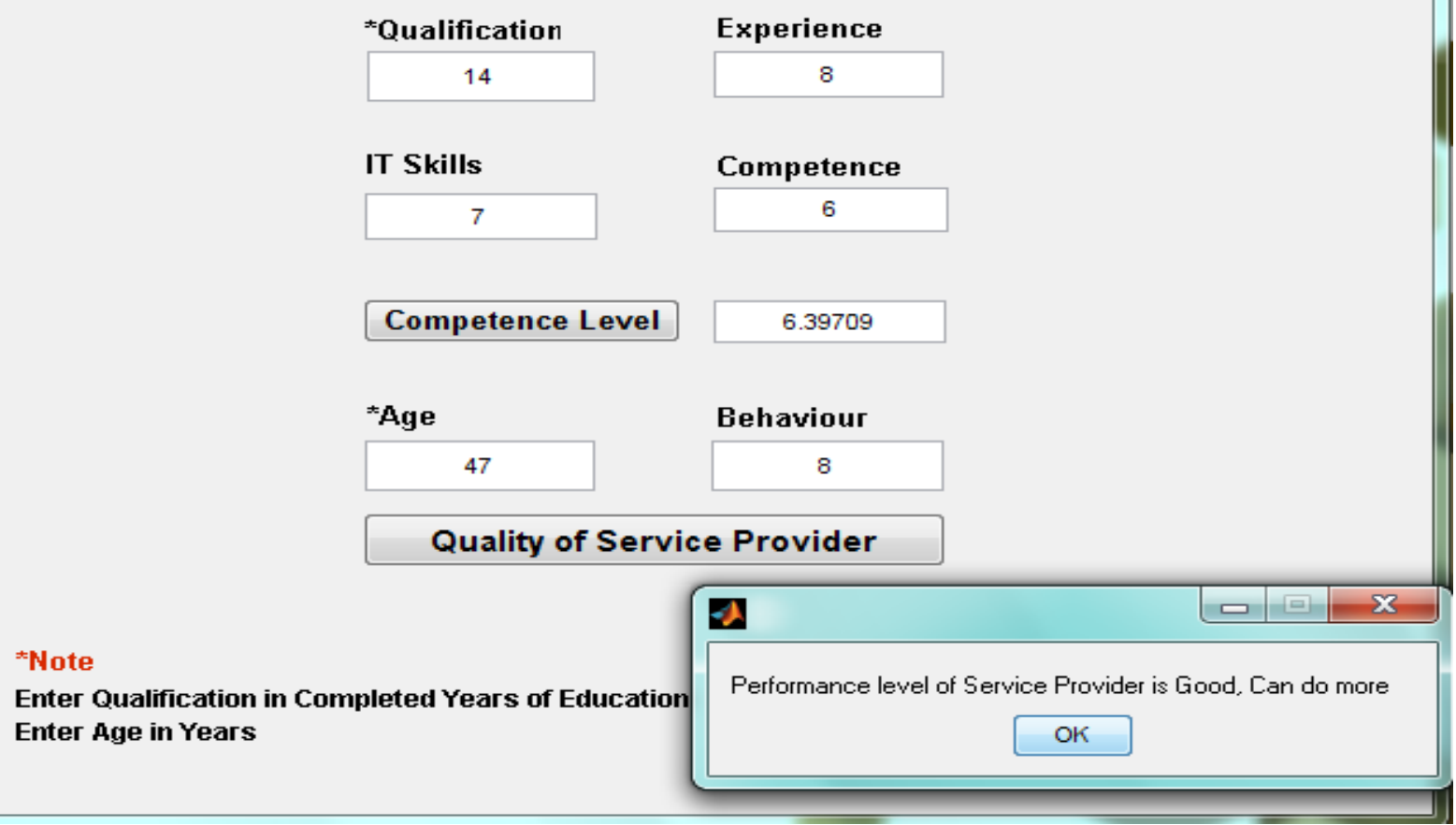

Fig 12: Graphical User Interface (GUI) for the Evaluation of Service Provider.

\section{Experiments and Outputs}

A few experiments were carried out with the Fuzzy Expert System model by changing the input variables values in the GUI designed specifically for FES which showed the change in the Quality of the Service Provider. The experiments which were carried out within the model were done on the basis of the changes made within the rule viewer by changing the input values of the FES model and observing the output selection of the categories for service providers.

\section{Conclusion and Future Work}

In this paper, a model has been developed based on fuzzy logic to evaluate the quality of service provided to the citizens by the service provider at the e-government center. A proper methodology was followed for the development of the Fuzzy Expert System and the results were validated within the real egovernment center environment. Our work is a clear solution to one of the major problems that need to be deeply considered while implementing e-government properly. The system clearly inclines the reliability to know the quality of service provider on the basis of well-defined factors. The results found by the developed system are compared with the data collected from the citizens. It has been found that the human experts in the field and the developed system produce similar results. The results from this expert system model will be used in further implementation of the model.

This paper discussed the design and development of the Fuzzy Expert System that incorporates critical factors for the quality evaluation of the service provided by the service provider in the e-government service center. In future, citizens will be categorized on the basis of their behavioral attributes along with demographic, experience and business value attributes. Once the categorization of the citizen is finished, a Fuzzy Expert System will be developed to assign any citizen to that of the pre-determined category of service providers. With this Fuzzy Expert System, we will be able to provide the service provider the minimum amount of information to be displayed on the screen to help the service provider to provide better services according to the need and background of the citizen.

Hence, the next step through this research is to develop Fuzzy Expert System to categorize the citizens coming to the egovernment service center to avail e-government services and to develop a framework which would identify the type of information required by the service provider to serve the citizen which will result increase in citizen satisfaction level. 


\section{REFERENCES}

[1] E-Government Handbook ,http://www.cdt.org/egov/ handbook/.

[2] Subhajyoti Ray and V. Venkata Rao "Evaluating Government Service: A customers' Perspective of egovernment"

[3] I.H. Osman, A.L. Anouze, Z. Irani, H .Lee, A. Balc1, T.D.Medeni, V. Weerakkody. 2011 "A new COBRAS framework to evaluate e-government services: a citizen centric perspective." CD-ROM/Online Proceedings of tGov Workshop'11 (1GOV11), Paper tGov-3, Brunel University, West London.

[4] Xenia Papadomichelaki, Babis Magoutas, Christos Halaris, Dimitris Apostolou, Gregoris Mentzas 2006" A review of quality dimensions in e-government services" Proceedings of the 5th international conference on Electronic Government(EGOV'06) Springer-Verlag.

[5] Jun Fei, Ruimin Yao, Lihua Yu 2008 "Fuzzy Analytic Hierarchy Process Application to E- government Performance Evaluation" Proceedings of IEEE Fifth International Conference on Fuzzy Systems and Knowledge Discovery.

[6] Babis Mgoutas, Gregoris Mentzas 2009 "Adaptive Evaluation of Portal Quality: An eGovernment Case". Proceedings of the 13th IEEE Panhellenic Conference on Informatics, pg 136-140.
[7] Piyush Gupta 2007 "Challenges and Issues in eGovernment Project Assessment" ACM ICEGOV, Macao.

[8] Gupta, M.P., Bhattacharya, J. and Agarwal, A. 2007 "Evaluating egovernment" University Press, 1-56.

[9] Marcin Sakowicz "How to Evaluate E-Government? Different Methodlogies and Methods"

[10] Babis Magoutas, Christos Halaris, and Gregoris Mentzas 2007 "An Ontology for the Multi-perspective Evaluation of Quality in E-Government Services" Springer-Verlag Berlin Heidelberg.

[11] Parmita Saha, Atanu Nath and Esmail Salehi-Sangari 2010 "Success of Government E -Service Delivery: Does Satisfaction Matter?" SpringerLink Lecture Notes in Computer Science, 2010, Volume 6228, 204-215

[12] http://www.mathworks.com/products/fuzzylogic

[13] Roy, R., Shah, S., Tiwari, A., and Hadden, J. 2006 "Soft Computing in the Service Industry", 6th Recent Advances in Soft Computing Conference.Canterbury, United Kingdom: Springer-Verlag. p 1-9.

[14] Wang YM, Yang YB, Xu DL, Chin KS 2006. On centroids of fuzzy numbers. Fuzzy Sets Syst., 157: 919926.

[15] Fuzzy Logic: Theory and Applications. Prentice Hall of India, New Delhi. 